\title{
Whole lung lavage using a rapid infusion system to treat a patient with pulmonary alveolar proteinosis
}

\author{
Seung Won Ra ${ }^{1}$, Soon Eun Park ${ }^{2}$, Hyung Kwan Lee ${ }^{2}$, Il Sang Han ${ }^{2}$, Se Hun Park ${ }^{2}$ \\ ${ }^{1}$ Department of Internal Medicine, Ulsan University Hospital, University of Ulsan College of Medicine, Ulsan, Korea \\ ${ }^{2}$ Department of Anesthesiology and Pain Medicine, Ulsan University Hospital, University of Ulsan College of Medicine, Ulsan, Korea
}

Received: August 21, 2019

Revised: October 1, 2019

Accepted: October 8, 2019

Corresponding author:

Se Hun Park

Department of Anesthesiology and

Pain Medicine, Ulsan University

Hospital, University of Ulsan College of

Medicine, 877 Bangeojinsunhwando-

ro, Dong-gu, Ulsan 44033, Korea

Tel: +82-52-250-7248

Fax: +82-52-251-8235

E-mail:nurunbab@uuh.ulsan.kr
Whole lung lavage $(\mathrm{WLL})$ is a therapeutic procedure to remove accumulated material by infusing and draining the lungs with lavage fluid. This procedure has been regarded as the current standard of care to treat pulmonary alveolar proteinosis. However, the WLL protocol has not yet been standardized and the technique has been refined and modified a number of times. A rapid infusion system is a device used to infuse blood or other fluids at precise rates and normothermic conditions. This device is not typically used in WLL, which relies on the passive infusion of fluids using the gravitational force. However, in this study we performed WLL using a rapid infusion system, since we aimed to take advantage of its shorter operation time and greater degree of control over fluid volume and temperature. The patient's symptoms improved without the occurrence of any complications.

Keywords: General anesthesia; Infusion pump; Lung lavage; Pulmonary alveolar proteinosis

\section{Introduction}

Pulmonary alveolar proteinosis (PAP) is a rare disorder that is characterized by accumulation of lipoproteinaceous material within alveoli, which impairs gaseous exchange and leads to progressive respiratory insufficiency [1]. PAP prevalence has been estimated to be in $0.37 / 100,000$ individuals [2]. Lipoproteinaceous material typically accumulate within the alveoli due to the impaired surfactant catabolism of alveolar macrophage [3]. Whole lung lavage (WLL) is widely practiced and currently used as a standard of care to physically remove the lipoproteinaceous material from the affected lung [4]. Recently, it has become common knowledge that the autoantibodies or abnormalities of granulocyte macrophage colony-stimulating factor (GM-CSF) is one of the major causes of PAP, which may be ameliorated by a medical treatment to supplement GM-CSF.
Of these treatment modalities, WLL is typically performed with the passive infusion of lavage fluid using the gravitational force. However, in a number of cases WLL was performed with a rapid infusion system [5], which is advantageous considering the fact that it can be used to adjust the volume of infused fluid while regulating the pressure applied during infusion. Additionally, it can heat the infusion fluid to help maintain the body temperature. We have focused on these advantages of a rapid infusion system and have reported here that a rapid infusion system was safely used in WLL.

\section{Case}

The patient provided written informed consent for publication of the research details and clinical images.

A 46-year-old man $166 \mathrm{~cm}$ tall and weighing $67 \mathrm{~kg}$, presented 
with symptoms of dyspnea after exercise for 5 months, but did not undergo treatment for the same. He had a history of smoking 20-packs of cigarettes per year without an underlying diseases. One week before visiting our hospital, he was treated with pneumonia specific antibiotics and steroids in another hospital. However, there was no improvement in his symptoms, following which he visited our hospital. He underwent a high-resolution computed tomography (HRCT) and transbronchial lung biopsy, all of which were consistent with the diagnosis of PAP. An HRCT of the chest revealed a ground glass opacity and an irregular crazy paving appearance in the left upper and right upper and lower lung fields (Fig. 1). On histopathologic pulmonary biopsy, no malignant cells were found, but the presence of amorphous proteinaceous substances in the lung alveoli was confirmed (Fig. 2).

At the time of diagnosis, arterial blood gas (ABG) analysis under room air revealed severe hypoxemia with $\mathrm{pH} 7.45$, arterial partial pressure of carbon dioxide $\left(\mathrm{PaCO}_{2}\right) 36.5 \mathrm{mmHg}$, arterial partial pressure of oxygen $\left(\mathrm{PaO}_{2}\right) 48.7 \mathrm{mmHg}$, bicarbonate

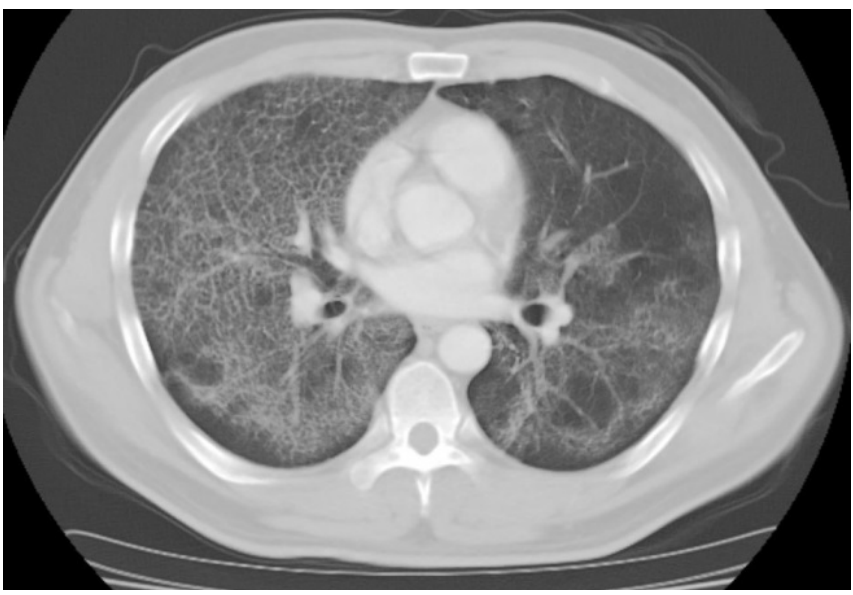

Fig. 1. Pre-whole lung lavage HRCT findings. HRCT scan shows ground glass opacities and crazy paving pattern. HRCT, highresolution computed tomography.
$\left(\mathrm{HCO}_{3}{ }^{-}\right) 25.1 \mathrm{mEq} / \mathrm{L}$, arterial saturation of oxygen $\left(\mathrm{SaO}_{2}\right) 86.6 \%$ (Table 1) and an alveolar arterial gradient $55.4 \mathrm{mmHg}$. Pulmonary function tests revealed a normal but decreased lung diffusing capacity, with a forced vital capacity (FVC) of $4.08 \mathrm{~L}$ (95\% of predicted value), a forced expiratory volume in 1 second $\left(\mathrm{FEV}_{1}\right)$ of $3.16 \mathrm{~L}$ ( $96 \%$ of predicted value), a ratio of $\mathrm{FEV}_{1} / \mathrm{FVC} 77 \%$, and diffusing capacity of the lungs for carbon monoxide of $57 \%$ of predicted value. We decided to perform therapeutic WLL considering the lack of improvement in the patient's clinical symptoms and hypoxemia. Based on the radiographic findings, we decided to first lavage the right lung, which seemed to be affected to a greater degree.

We administered oxygen $(6 \mathrm{~L} / \mathrm{min})$ via a nasal cannula. The patient had entered the operation room while appearing to be mentally alert and subsequently, electrodes from an electrocardiogram, pulse oximeter, noninvasive blood pressure (BP) monitor, and bispectral index (BIS) monitor were attached to the patient. The radial artery catheter was placed under local anesthesia to

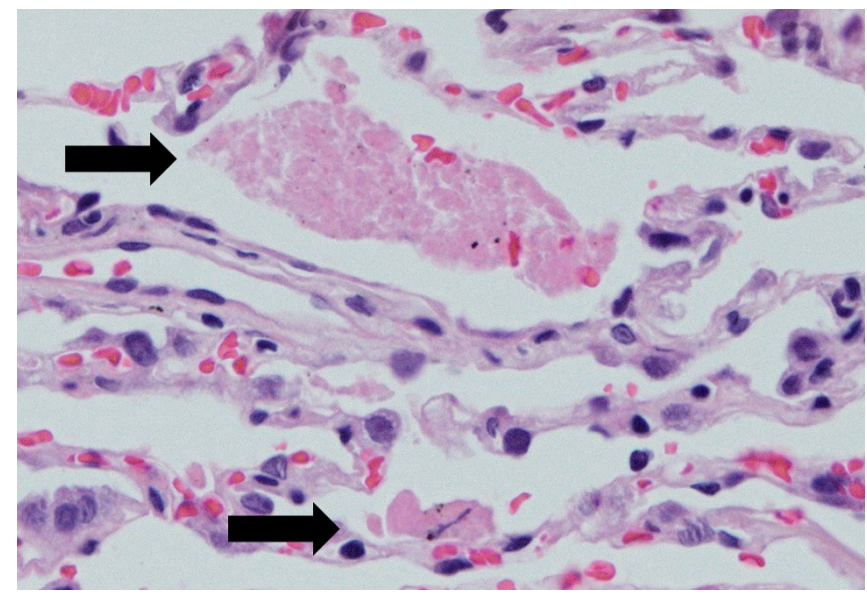

Fig. 2. Transbronchial lung biopsy specimen from right lower lobe shows intraalveolar pinkish proteinaceous material (arrows), consistent with pulmonary alveolar proteinosis (hematoxylin and eosin stain, $\times 400)$.

Table 1. Arterial blood gas analysis

\begin{tabular}{lccccc}
\hline & $\begin{array}{c}\text { Admission } \\
\left(\mathrm{FiO}_{2} \mathrm{0.2}\right)\end{array}$ & $\begin{array}{c}\text { Before induction of anesthesia })^{\text {a }} \\
\left(\mathrm{FiO}_{2} \mathrm{0.44}\right)\end{array}$ & $\begin{array}{c}\text { After lung isolation } \\
\left(\mathrm{FiO}_{2} 1.0\right)\end{array}$ & $\begin{array}{c}\text { After lavage } \\
\left(\mathrm{FiO}_{2} 0.45\right)\end{array}$ & $\begin{array}{c}\text { Discharge } \\
\left(\mathrm{FiO}_{2} 0.2\right)\end{array}$ \\
\hline $\mathrm{pH}$ & 7.45 & 7.44 & 7.37 & 7.41 & 7.39 \\
$\mathrm{PaCO}_{2}(\mathrm{mmHg})$ & 36.5 & 35.0 & 38.0 & 32.0 & 38.7 \\
$\mathrm{PaO}_{2}(\mathrm{mmHg})$ & 48.7 & 58.0 & 84.0 & 103.0 & 87.2 \\
$\mathrm{HCO}_{3}^{-}(\mathrm{mEq} / \mathrm{L})$ & 25.1 & 23.8 & 22.0 & 20.3 & 22.8 \\
$\mathrm{SaO}_{2}(\%)$ & 86.6 & 91.0 & 96.0 & 98.0 & 96.1 \\
\hline
\end{tabular}

$\mathrm{FiO}_{2}$, fraction of inspired oxygen; $\mathrm{PaCO}_{2}$, arterial partial pressure of carbon dioxide; $\mathrm{PaO}_{2}$, arterial partial pressure of oxygen; $\mathrm{HCO}_{3}^{-}$, bicarbonate; SaO arterial saturation of oxygen.

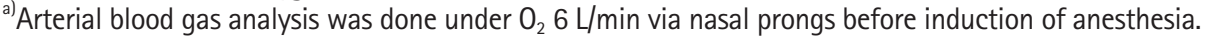


continuously monitor any changes in $\mathrm{BP}$ and intermittently analyze changes in ABG - before inducing anesthesia. Pulse oxygen saturation was $91 \%$ in the room before inducing anesthesia, and ABG analysis revealed $\mathrm{pH} 7.44, \mathrm{PaCO}_{2} 35.0$ mmHg, $\mathrm{PaO}_{2} 58.0$ $\mathrm{mmHg}, \mathrm{HCO}_{3}^{-} 23.8 \mathrm{mEq} / \mathrm{L}$, and $\mathrm{SaO}_{2} 91 \%$ (Table 1). Anesthesia was induced with $300 \mathrm{mg}$ of $2.5 \%$ pentothal sodium, $60 \mathrm{mg}$ of $2 \%$ lidocaine, and $50 \mathrm{mg}$ of rocuronium. After a 3-minute manual ventilation, the left mainstem bronchus was uneventfully intubated with a 37-Fr left-sided double-lumen endotracheal tube (Broncho-Cath ${ }^{\circledast}$ Mallinckrodt, St. Louis, MO, USA), which was confirmed by a flexible bronchoscope. The cuffs were inflated with just enough air to ensure proper lung isolation and adequate ventilation without a leak. A central venous catheter was inserted into the right internal jugular vein and an esophageal stethoscope temperature sensor was inserted through the oral cavity.

Both lungs were ventilated with $100 \%$ oxygen for 10 minutes to denitrogenate them, and we commenced the single lung ventilation of the left lung to absorb oxygen in the right lung. We controlled sevoflurane concentration to maintain the BIS between 40 and 60 during the operation and monitored the change in pulse oxygen saturation, supplying $100 \%$ oxygen at a flow rate of $3 \mathrm{~L} /$ min through a mechanical ventilator. $\mathrm{ABG}$ analysis after lung isolation revealed $\mathrm{pH}$ 7.37, $\mathrm{PaCO}_{2} 38.0 \mathrm{mmHg}, \mathrm{PaO}_{2} 84.0 \mathrm{mmHg}$, $\mathrm{HCO}_{3}{ }^{-} 22.0 \mathrm{mEq} / \mathrm{L}$, and $\mathrm{SaO}_{2} 96 \%$ (Table 1).

We changed the patient's position to left lateral decubitus with the right lung positioned above the other, and re-confirmed the position of the tube with a flexible bronchoscope. A tube attached to the lower limb of a Y-connector was connected to the right side of the double lumen endotracheal tube while the patient's pulse oxygen saturation under lung isolation was continuously monitored. A tube attached to the upper left limb of the a Y-connector was connected to a rapid infusion system (FMS2000 ${ }^{\mathrm{rm}}$, Belmont Instrument Co., Billerica, MA, USA) to commence infusion and a tube attached to the upper right limb was connected to the drain tube under the surgical bed (Fig. 3). Two upper tubes had clamps to control the flow of the fluid in and out of the right lung. A pressure monitoring device was placed on the infusion tube so that the infusion pressure could be monitored in real time. The initial aliquot of $1,000 \mathrm{~mL}$ normal saline, warmed to $37.5^{\circ} \mathrm{C}$, was infused into the lung at a rate of $150 \mathrm{~mL} / \mathrm{min}$ using a rapid infusion system and was subsequently drained. This process was repeated till the drainage solution was adequately clear, which took a total of 10 cycles. During this process, an assistant performed manual chest percussion, and the remnant fluid was aspirated after the last drainage. Total infusion/drainage was $10,000 \mathrm{~mL} / 9,250 \mathrm{~mL}$, and the total lavage time was 2 hours. After lung lavage, the patient was moved into a supine position and was transferred to the intensive care unit (ICU) after exchanging a double-lumen endotracheal tube for a single lumen endotracheal tube.

In the ICU, the patient was provided to ensure pressure controlled mechanical ventilation with the pressure of $14 \mathrm{mmHg}$, an inhaled oxygen concentration of 0.45 and respiratory rate of 18 breaths/min. ABG analysis revealed $\mathrm{pH} 7.41, \mathrm{PaCO}_{2} 32.0 \mathrm{mmHg}$, $\mathrm{PaO}_{2} 103.0 \mathrm{mmHg}, \mathrm{HCO}_{3}^{-} 20.3 \mathrm{mEq} / \mathrm{L}$, and $\mathrm{SaO}_{2} 98 \%$ (Table 1). The patient was extubated 4 hours after ICU admission, and pulse oxygen saturation was maintained at $95 \%$ or more under administration of oxygen $(4 \mathrm{~L} / \mathrm{min})$ via a nasal cannula. Four days later, the left lung was treated with the same procedure. After the clinical symptoms improved, he was discharged after 1 week. ABG analysis that was performed right before the patient's discharge revealed $\mathrm{pH}$ 7.39, $\mathrm{PaCO}_{2} 38.7 \mathrm{mmHg}, \mathrm{PaO}_{2} 87.2 \mathrm{mmHg}, \mathrm{HCO}_{3}$ $22.8 \mathrm{mEq} / \mathrm{L}$, and $\mathrm{SaO}_{2} 96.1 \%$ (Table 1).

\section{Discussion}

PAP was first described by Rosen et al. [6] in 1958, since the accumulation of lipoproteinaceous material in the alveoli lead to nonspecific symptoms such as cough, hypoxemia, and dyspnea. Histopathologic examination reveals that presence of lipoproteinaceous material in the alveoli. Radiographic appearance of PAP are extensive bilateral infiltration on chest radiograph and groundglass opacifications which is commonly referred to as crazy paving on computed tomography images. However, there is often a dissociation between the extent of radiographic abnormalities and severity of the symptoms and physical findings [1], and variable/ nonspecific clinical symptoms delay PAP diagnosis [7]. PAP has been classified into three types according to the developmental

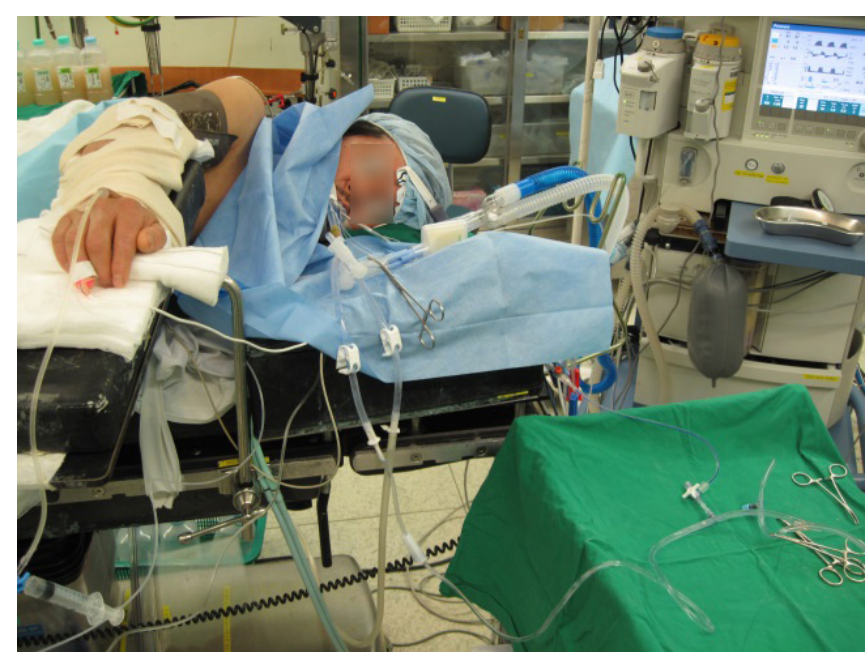

Fig. 3. Left lateral decubitus position (lavage side up) and application of a Y-connector attached to the double lumen endotracheal tube. 
mechanism: congenital, idiopathic, and secondary. Idiopathic PAP mentioned in this study is considered to represent approximately $90 \%$ of PAP cases. According to Inoue et al. [8] in 2008, the male to female ratio was $2.1: 1$, the median age at diagnosis was 51 years, and a history of smoking occurred in $56 \%$.

The pathogenesis of PAP has remained unclear for several decades after its first report. However, a report in 1994 that used knockout mice which were deficient in GM-CSF gene demonstrated similar pulmonary pathology as human PAP and subsequently provided a fresh perspective on the causes of the disease [9]. PAP results from an accumulation of surfactant that appeared to occur due to its impaired clearance and not its overproduction [10]. In addition, autoantibody against GM-CSF was found to be present in all specimens of bronchoalveolar lavage fluid obtained from 11 idiopathic PAP patients [11]. Therefore, it is now accepted that dysfunction of alveolar macrophages caused by abnormalities of GM-CSF due to autoantibodies leads to the condition of idiopathic PAP. This finding has been associated with medical treatment modalities that include exogenous GM-CSF [12].

These novel therapeutic modalities for PAP have prompted the necessity of evaluating the efficacy of WLL, which was presented by Ramirez et al. [13] in 1963 as the primary treatment modality. According to a study by Gay et al. [14] in 2017, the effectiveness of WLL was demonstrated by the improvement of short-term clinical symptoms, including arterial partial pressure oxygen after lavage although the application of the WLL technique was variable. The use of exogenous GM-CSF supplementation as the first line of therapeutic intervention has its limitations due to a lack of placebo-controlled randomized trials with large sample sizes [15]. Additionally, randomized trials that have compared WLL with exogenous GM-CSF supplementation therapies have not yet been performed [15]. Therefore, we decided to perform WLL first.

The primary indications for WLL include decreased pulmonary function, decreased arterial oxygen saturation at rest, and abnormal radiographic findings. All of these were present in this case [16]. Complications that may occur during WLL are fever, hypoxemia, pneumonia, and pneumothorax, all of which were absent in this case. Due to a lack of a standardized protocol, there are various aspects of the procedure which function under the premise of physically removing the accumulated lipoproteinaceous material by washing the alveoli. However, in most institutions, lavage is performed under general anesthesia with lung isolation [17]. There are several variations considering the anesthetic maintenance agent, the choice of lung to be lavaged, the patient's position at the time of lavage, the volume of lavage fluid, and the method of chest percussion [16].

Here, the right lung appeared to be in a poorer condition on the radiographic findings was lavaged first, and left lung lavage was followed after 4 days. The patient was positioned in the lateral decubitus position and manual chest percussions were performed by an assistant during the lavage. Infusing fluid by gravity is widely selected method among institutions. However, we adopted a rapid infusion system which has an advantage of shortening lavage time and maintaining constant temperature and pressure; furthermore, decreases labor intensity by reducing the need for fluid replacement, controlling accurate infusion rate in real time, and measuring the amount of infused fluid accurately [18]. Although it was possible to perform bilateral sequential lung lavage in one session, we performed a single lung lavage in one session. Since this was the first time that we adopted a rapid infusion system for WLL, we had to consider possible adverse effect of using a rapid infusion system for WLL.

Possible adverse effect of using a rapid infusion system could be caused by barotrauma. Observing the infusing pressure in real time seemed to be helpful. In practice, it is recommended to use a liquid bag placed $50 \mathrm{~cm}$ above the thoracic cavity [17], for which the pressure would be equal to $36.78 \mathrm{mmHg}$. The average pressure observed in this case was $22 \mathrm{mmHg}$ at the time of infusion (Fig. 4). Barotrauma caused by a rapid infusion system was not observed in clinical and radiological aspects. The use of a rapid infusion system resulted in a shorter procedural time than other cases which used similar amount of fluid [19].

There is no consensus regarding the total volume of infusion in WLL, and it varied significantly 5-40 liters of saline [16]. Most institutions repeat the infusion and drainage until the effluent is relatively clear by visual inspection. In a previous case of using a

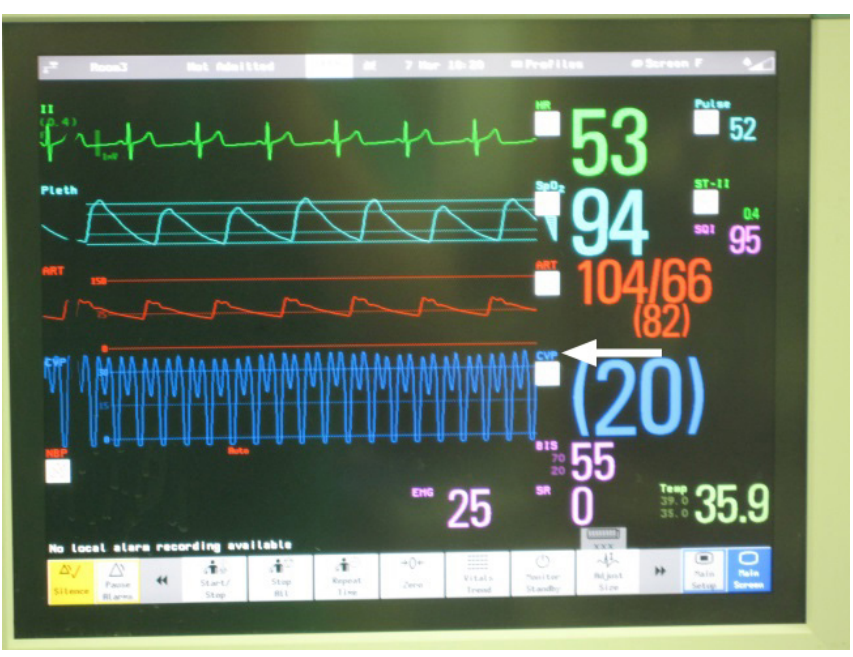

Fig. 4. Intraoperative monitored vital sign denoted as 'central venous pressure' (arrow) shows pressure of injected fluid to the right lung. 
rapid infusion system for WLL, 8.5-16 liters of normal saline was used [5]. In our opinion, a rapid infusion system will help to shorten operation time when relatively large volume of infusion is needed. However, further reports and studies are needed to establish the cutoff volume of infusion in using a rapid infusion system for WLL.

In this case, the discordance of the volume of infusion and drainage might suggest leakage into the contralateral lung. However, it was our understanding that leakage did not occur in regards of not quantifying the amount of last aspiration fluid, achieving successful lung isolation; furthermore, there was no evidence or suspicion of leakage, which presented by a desaturation on the monitoring equipment. In addition, there were no post procedural complications such as respiratory insufficiency and hypoxemia, which might occur when the fluid had leaked [20]. The average pressure at the time of infusion was low when using a rapid infusion system; however, it should be considered that there is a possibility of using high pressure since the difference in pressure depends on the characteristics of the rapid infusion system.

WLL is performed as the main treatment modality for PAP, but the treatment method needs to be standardized and various methods are used. In conclusion, we successfully performed WLL with a rapid infusion system which has several advantages such as: shortening the lavage time, maintaining constant temperature and pressure without adverse effects caused by barotrauma.

\section{Acknowledgments}

\section{Conflicts of interest}

No potential conflict of interest relevant to this article was reported.

\section{ORCID}

Seung Won Ra, https://orcid.org/0000-0002-2458-8414

Soon Eun Park, https://orcid.org/0000-0002-7184-2067

Hyung Kwan Lee, https://orcid.org/0000-0002-1504-8934

Il Sang Han, https://orcid.org/0000-0002-6475-9310

Se Hun Park, https://orcid.org/0000-0003-2264-4627

\section{References}

1. Trapnell BC, Whitsett JA, Nakata K. Pulmonary alveolar proteinosis. N Engl J Med 2003;349:2527-39.

2. Ben-Dov I, Kishinevski Y, Roznman J, Soliman A, Bishara H, Zelligson E, et al. Pulmonary alveolar proteinosis in Israel: ethnic clustering. Isr Med Assoc J 1999;1:75-8.

3. Trapnell BC, Whitsett JA. Gm-CSF regulates pulmonary sur- factant homeostasis and alveolar macrophage-mediated innate host defense. Annu Rev Physiol 2002;64:775-802.

4. Luisetti M, Kadija Z, Mariani F, Rodi G, Campo I, Trapnell BC. Therapy options in pulmonary alveolar proteinosis. Ther Adv Respir Dis 2010;4:239-48.

5. Moy EK, Pistun O, Teba C, Jagpal S, Hussain S. A rapid infuser system for whole-lung lavage. J Bronchology Interv Pulmonol 2016;23:e6-8.

6. Rosen SH, Castleman B, Liebow AA. Pulmonary alveolar proteinosis. N Engl J Med 1958;258:1123-42.

7. Huizar I, Kavuru MS. Alveolar proteinosis syndrome: pathogenesis, diagnosis, and management. Curr Opin Pulm Med 2009; 15:491-8.

8. Inoue Y, Trapnell BC, Tazawa R, Arai T, Takada T, Hizawa N, et al. Characteristics of a large cohort of patients with autoimmune pulmonary alveolar proteinosis in Japan. Am J Respir Crit Care Med 2008; 177:752-62.

9. Stanley E, Lieschke GJ, Grail D, Metcalf D, Hodgson G, Gall JA, et al. Granulocyte/macrophage colony-stimulating factor-deficient mice show no major perturbation of hematopoiesis but develop a characteristic pulmonary pathology. Proc Natl Acad Sci U S A 1994;91:5592-6.

10. Ikegami M, Ueda T, Hull W, Whitsett JA, Mulligan RC, Dranoff $\mathrm{G}$, et al. Surfactant metabolism in transgenic mice after granulocyte macrophage-colony stimulating factor ablation. Am J Physiol 1996;270:L650-8.

11. Kitamura T, Tanaka N, Watanabe J, Uchida, Kanegasaki S, Yamada $\mathrm{Y}$, et al. Idiopathic pulmonary alveolar proteinosis as an autoimmune disease with neutralizing antibody against granulocyte/macrophage colony-stimulating factor. J Exp Med 1999;190:875-80.

12. Seymour JF, Dunn AR, Vincent JM, Presneill JJ, Pain MC. Efficacy of granulocyte-macrophage colony-stimulating factor in acquired alveolar proteinosis. N Engl J Med 1996;335:1924-5.

13. Ramirez J, Schultz RB, Dutton RE. Pulmonary alveolar proteinosis: a new technique and rationale for treatment. Arch Intern Med 1963;112:419-31.

14. Gay P, Wallaert B, Nowak S, Yserbyt J, Anevlavis S, Hermant C, et al. Efficacy of whole-lung lavage in pulmonary alveolar proteinosis: a multicenter international study of GELF. Respiration 2017;93:198-206.

15. Kumar A, Abdelmalak B, Inoue Y, Culver DA. Pulmonary alveolar proteinosis in adults: pathophysiology and clinical approach. Lancet Respir Med 2018;6:554-65.

16. Campo I, Luisetti M, Griese M, Trapnell BC, Bonella F, Grutters J, et al. Whole lung lavage therapy for pulmonary alveolar proteinosis: a global survey of current practices and procedures. 
Orphanet J Rare Dis 2016;11:115.

17. Awab A, Khan MS, Youness HA. Whole lung lavage-technical details, challenges and management of complications. J Thorac Dis 2017;9:1697-1706.

18. Mccunn M, Grissom TE, Dutton RP. Anesthesia for trauma. In: Miller RD, Cohen NH, Eriksson LI, Fleisher LA, Wiener-Kronish JP, Young WL, editors. Miller's anesthesia. 8th ed. Philadel- phia: Elsevier/Saunders; 2015. p. 2423-59.

19. Lee JH, Chung CJ, Oh JN, Ko BJ, Choi SR. Anesthetic management of whole-lung lavage using propofol-remifentanil in a patient with pulmonary alveolar proteinosis. Korean J Crit Care Med 2012;27:173-8.

20. Ramachandran P, Chaudhury A, Devaraj U, Maheshwari KU, D'Souza G. Monitoring whole-lung lavage using lung ultrasound: the changing phases of the lung. Lung India 2018; $35: 350-3$. 